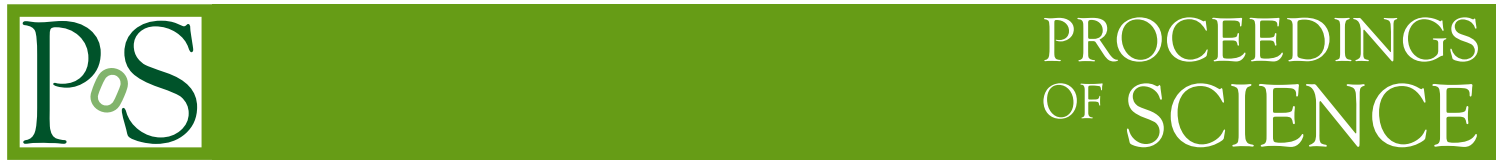

\title{
Direct Photon Results from Tevatron
}

\author{
Costas Vellidis*† \\ Fermilab \\ E-mail: vellidisefnal.gov
}

\begin{abstract}
This article presents recent measurements of differential cross sections for single and double direct photon production from the $\mathrm{CDF}$ and $\mathrm{D} 0$ experiments running at the Tevatron. For single photon production, results are shown for both the inclusive and the exclusive production in association with jets. Comparisons of the measurements with next-to-leading order perturbative QCD calculations show good agreement for the inclusive single photon production at high energies and for the diphoton production in the range of kinematics relevant to Higgs searches. The agreement is poor at kinematic regions where soft gluon interactions and fragmentations are important. For the exclusive single photon production the agreement is generally poor.
\end{abstract}

35th International Conference of High Energy Physics

July 22-28, 2010

Paris, France

\footnotetext{
* Speaker.

${ }^{\dagger}$ On behalf of the CDF and D0 Collaborations.
} 


\section{Introduction}

Measurements of differential cross sections for the production of high transverse momentum $p_{T}^{\gamma}$ "prompt" or "direct" photons, i.e. photons not coming from neutral hadron decays or from radiation in the detector material, are a precision probe for understanding the dynamics of high energy hadron collisions and for searching new phenomena. Fermilab Tevatron is an ideal place to conduct such measurements. It is a highly performing $p \bar{p}$ collider, operating at $\sqrt{s}=1.96 \mathrm{TeV}$, which has delivered so far $\sim 9 \mathrm{fb}^{-1}$ of integrated lminosity $\left(\sim 7 \mathrm{fb}^{-1}\right.$ currently on tape for each experiment). It is equipped with two well understood detectors, CDF and D0, instrumented with high resolution electromagnetic calorimeters, which can measure photons with high precision.

\section{Single Photon Production}

Measurements of the single photon production cross section test perturbative QCD (pQCD) with potential to constrain the parton distribution functions (PDF) of the proton. Quark annihilation, $q \bar{q} \rightarrow \gamma g$, and Compton scattering, $g q \rightarrow \gamma q$ or $g q \rightarrow g q$ where the final state quark fragments to a photon, dominate in leading order (LO). Figure 1 shows on the left the inclusive cross section measured at $\mathrm{CDF}$ [1] as a function of $p_{T}^{\gamma}$ in comparison with a next-to-leading order (NLO) pQCD calculation [2]. The ratios of the exclusive $\gamma+$ jet cross section measured at D0 [3] to the NLO pQCD predictions [2] as functions of $p_{T}^{\gamma}$ are shown on the right in four angular regions, with a central or forward jet close to or away from the photon, to separate between low and high $x$ parton scattering. The theory agrees well with the inclusive measurement, except at $p_{T}^{\gamma}<50 \mathrm{GeV} / \mathrm{c}$ where fragmentations become important. The agreement is poor for the exclusive cross section.
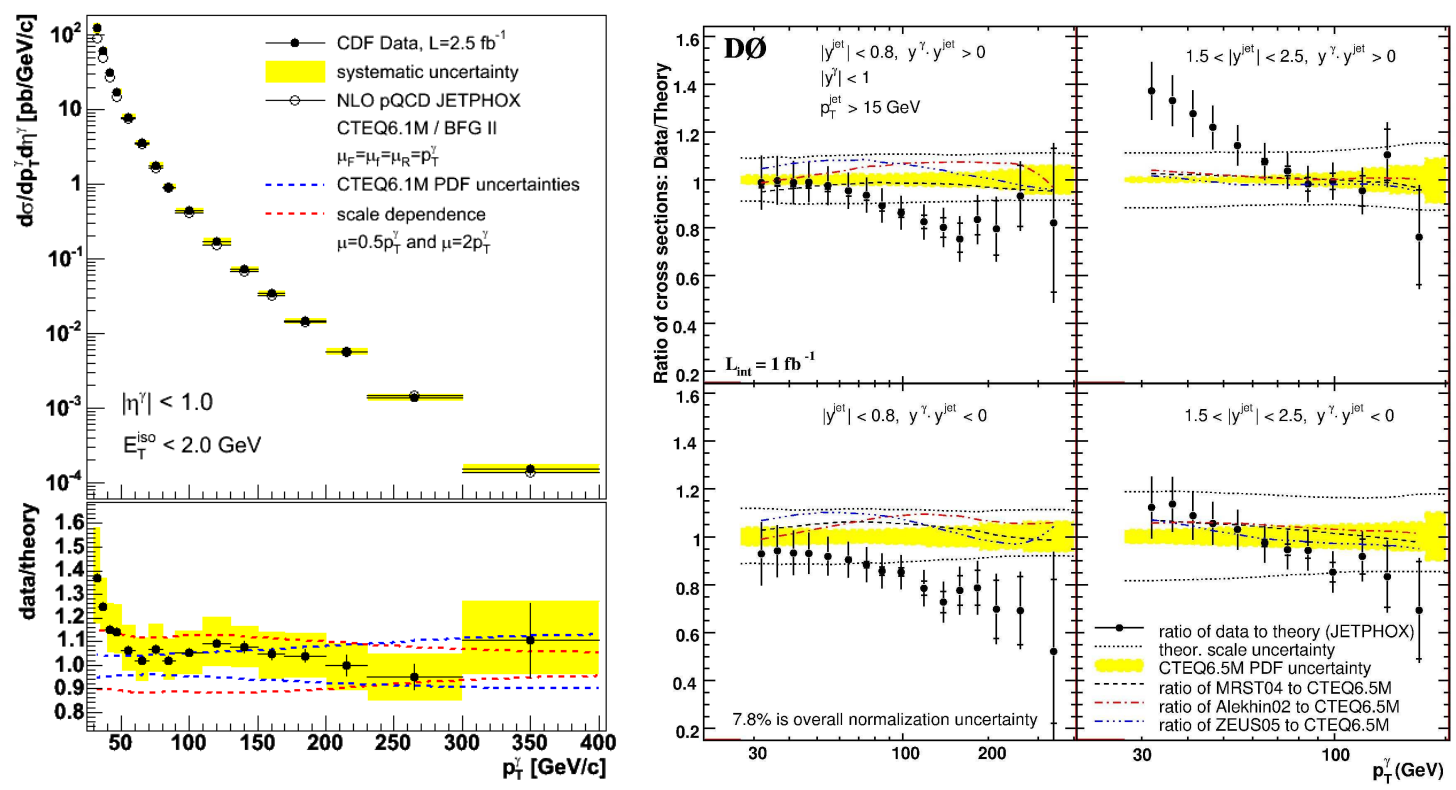

Figure 1: Left: $p \bar{p} \rightarrow \gamma+X$ cross section vs. the photon $p_{T}$ measured with the CDF detector. Right: Ratio of data to theory vs. the photon $p_{T}$ for the $p \bar{p} \rightarrow \gamma+$ jet $+X$ cross section measured with the D0 detector. 


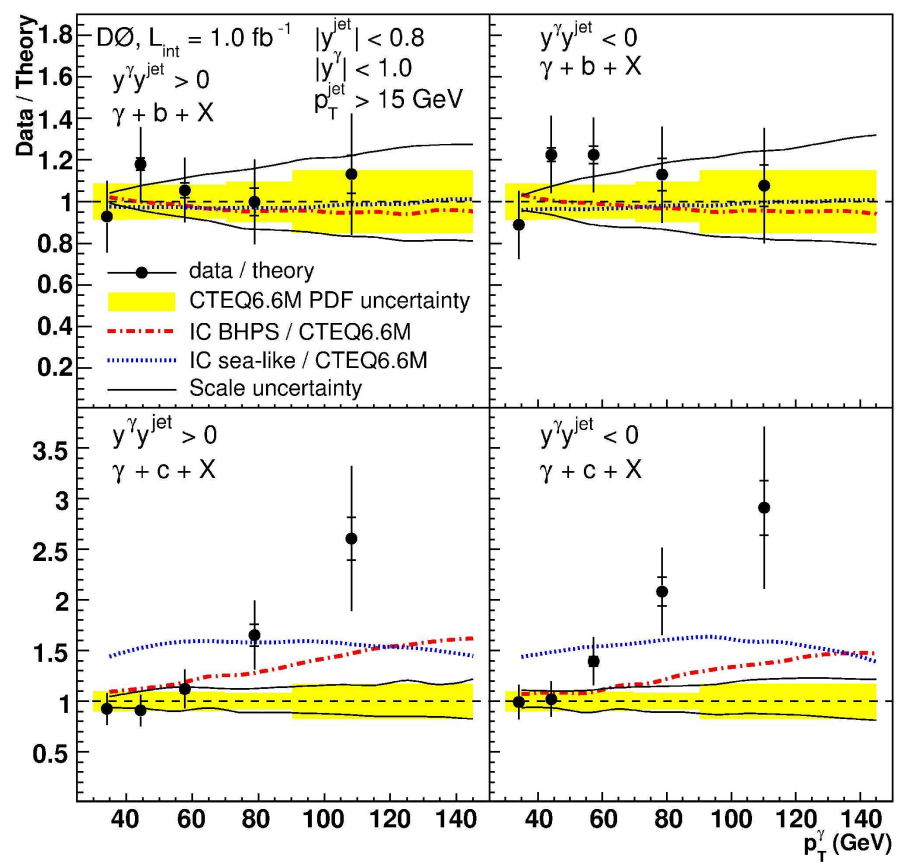

Figure 2: Ratio of data to theory vs. the photon $p_{T}$ for the $p \bar{p} \rightarrow \gamma+b-, c-$ jet $+X$ cross section measured with the D0 detector.

Of special interest is the measurement of the exclusive $\gamma+$ jet cross section where the associated jet arises from a heavy flavor ( $b$ or $c$ ) quark, which tests the heavy flavor and gluon contents of the proton. Figure 2 shows the ratios of this cross section measured at D0 [4] to NLO pQCD predictions [5] as functions of $p_{T}^{\gamma}$, again in four angular regions. The agreement is good for the $b$ flavor, as observed in a CDF measurement [6] too, but poor for the $c$ flavor, with a slight improvement by adding intrinsic charm (IC) flavor to the CTEQ6.6M PDF set [7] used in the calculation.

\section{Diphoton Production}

Diphoton production is a channel where searches for light mass Higgs and new phenomena, such as heavy resonances and extra spatial dimensions, are conducted or planned. Direct $\gamma \gamma$ production is an irreducible background to these searches which needs to be understood. Measurements of $\gamma \gamma$ production cross sections differential in various kinematic variables also test pQCD. Quark annihilation, $q \bar{q} \rightarrow \gamma \gamma$, gluon fusion, $g g \rightarrow \gamma \gamma$, and Compton scattering, $g q \rightarrow \gamma \gamma q$, dominate in LO. Fragmentations also play a role in the gluon fusion and Compton scattering channels.

Diphoton production cross sections measured at D0 [8] and CDF [9] are compared in Figure 3 with LO [10] and NLO [11, 12] calculations. The NLO predictions are in good agreement with the data in the diphoton invariant mass spectrum (left), with a cut on $M_{\gamma \gamma}>p_{T}^{\gamma \gamma}$ imposing kinematics similar to low mass Higgs $\rightarrow \gamma \gamma$ decays. However, they underestimate the data in the spectrum of the azimuthal difference $\Delta \phi$ of the photon pair (right), especially at low $\Delta \phi$ where soft gluon scattering and fragmentations are expected to contribute significantly. 

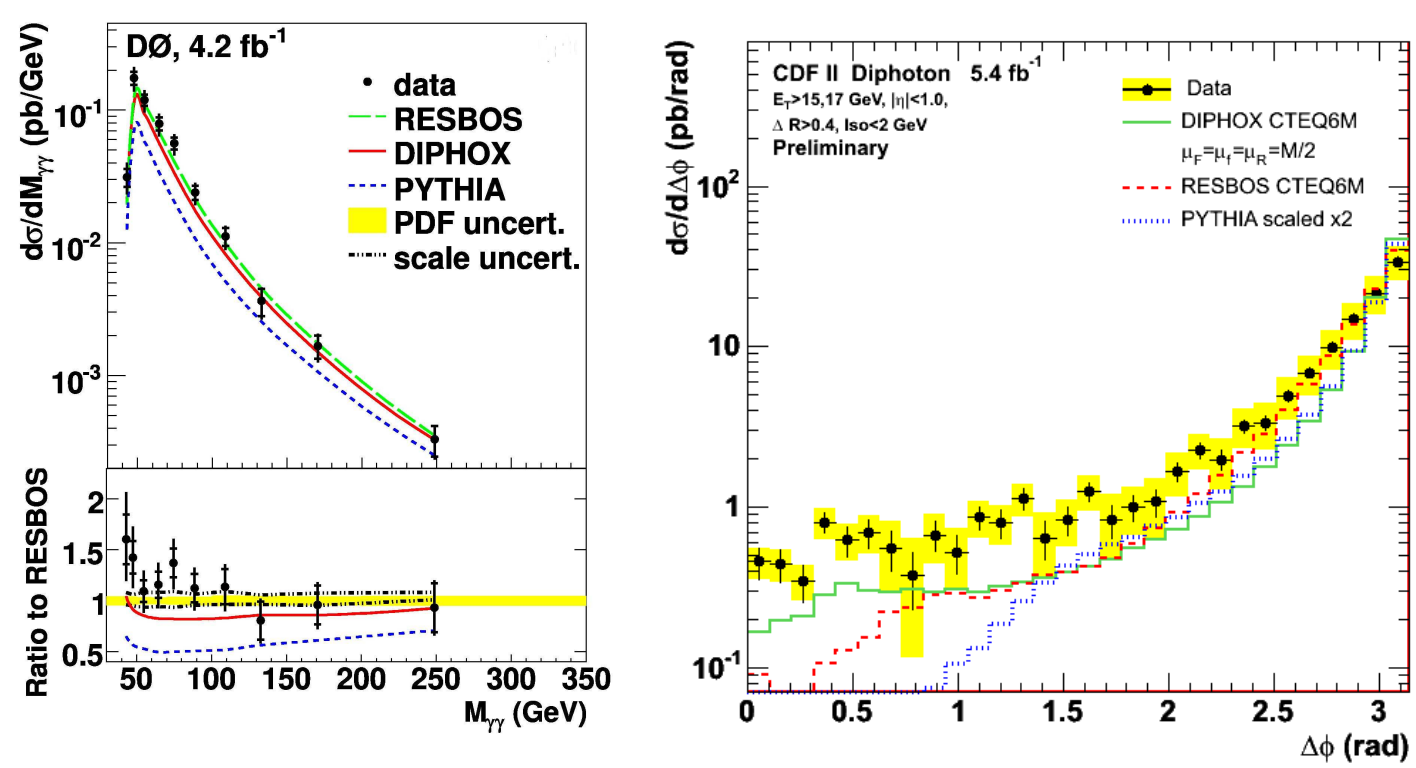

Figure 3: Left: $p \bar{p} \rightarrow \gamma \gamma+X$ cross section vs. the diphoton invariant mass for $M_{\gamma \gamma}>p_{T}^{\gamma \gamma}$ measured with the D0 detector. Right: $p \bar{p} \rightarrow \gamma \gamma+X$ cross section vs. the diphoton azimuthal difference $\Delta \phi=\left|\phi_{\gamma 1}-\phi_{\gamma 2}\right| \bmod \pi$ measured with the CDF detector.

\section{Summary}

High precision measurements of differential cross sections for direct photon production over wide kinematic ranges have been recently published from both the D0 and CDF Collaborations. Single direct photon production cross sections have been measured for inclusive production, $\gamma+$ jet exclusive production and $\gamma+$ heavy flavor jet production. NLO pQCD calculations do not describe well the exclusive production and fragmentation sensitive distributions of the diphoton production.

\section{References}

[1] T. Aaltonen et al. (CDF Collaboration), Phys. Rev. D 80, 111106 (2009); arXiv:0910.3623v2.

[2] S. Catani et al., JHEP 0205, 028 (2002).

[3] V. M. Abazov et al. (D0 Collaboration), Phys. Lett. B 666, 2435 (2008); arXiv.org:0804.1107.

[4] V. M. Abazov et al. (D0 Collaboration), Phys. Rev. Lett. 102, 192002 (2009); arXiv.org:0901.0739.

[5] B. W. Harris et al., Phys. Rev. D 65, 094032 (2002); arXiv:0901.3791v1.

[6] T. Aaltonen et al. (CDF Collaboration), Phys. Rev. D 81, 052006 (2010); arXiv:0912.3453.

[7] J. Pumplin et al., Phys. Rev. D 75, 054029 (2007).

[8] V. M. Abazov et al. (D0 Collaboration), Phys. Lett. B 690, 108 (2010); arXiv.org:1002.4917.

[9] www-cdf.fnal.gov/physics/new/qcd/diphXsec_2010/public_diphoton.html

[10] T. Sjöstrand et al., JHEP 0605, 026 (2006).

[11] T. Binoth et al., Eur. Phys. J. C 16, 311 (2000).

[12] C. Balazs et al., Phys. Rev. D 76, 013009 (2007). 